\title{
Analysis of the human interaction with a wearable lower-limb exoskeleton
}

\author{
Juan C. Moreno ${ }^{\mathrm{a} *}$, Fernando Brunetti ${ }^{\mathrm{a}}$, Enrique Navarro ${ }^{\mathrm{b}}$, Arturo Forner-Cordero ${ }^{\mathrm{a}}$ and José L. Pons ${ }^{\mathrm{a}}$ \\ ${ }^{a}$ Grupo de Bioingeniería, IAI, Consejo Superior de Investigaciones Cientificas, Arganda del Rey, Madrid, España; ${ }^{b}$ Facultad de la \\ Actividad Física y el Deporte (INEF), Universidad Politécnica de Madrid. Martín Fierro s/n, Madrid, España
}

(Received 13 October 2008; final version received 16 February 2009)

\begin{abstract}
The design of a wearable robotic exoskeleton needs to consider the interaction, either physical or cognitive, between the human user and the robotic device. This paper presents a method to analyse the interaction between the human user and a unilateral, wearable lower-limb exoskeleton. The lower-limb exoskeleton function was to compensate for muscle weakness around the knee joint. It is shown that the cognitive interaction is bidirectional; on the one hand, the robot gathered information from the sensors in order to detect human actions, such as the gait phases, but the subjects also modified their gait patterns to obtain the desired responses from the exoskeleton. The results of the two-phase evaluation of learning with healthy subjects and experiments with a patient case are presented, regarding the analysis of the interaction, assessed in terms of kinematics, kinetics and/or muscle recruitment. Human-driven response of the exoskeleton after training revealed the improvements in the use of the device, while particular modifications of motion patterns were observed in healthy subjects. Also, endurance (mechanical) tests provided criteria to perform experiments with one post-polio patient. The results with the post-polio patient demonstrate the feasibility of providing gait compensation by means of the presented wearable exoskeleton, designed with a testing procedure that involves the human users to assess the human-robot interaction.
\end{abstract}

Keywords: biological motor systems; man-machine systems; robots; exoskeletons

\section{Background}

Biomechatronics is the application of mechatronics (study, analysis, design and implementation of hybrid systems comprising mechanical, electrical and control components or subsystems) to biological motor systems (Carrozza et al. 2002; Pons 2008; Pons et al. 2008). One outstanding biomechatronic application is the design of robots to improve human performance and, more specifically, for rehabilitation. Wearable robots involve a wide range of devices that provide functional enhancement of healthy users as well as compensation and therapy for the disabled. From a robotics perspective, exoskeletons are mechatronic devices in which their segments and joints have a correspondence and are coupled with those of the human body (Rocon 2005). Robotic exoskeletons are used in different rehabilitation applications that can be classified under several categories (Harwin et al. 2002). For instance, they can serve as posture-support mechanisms. They can be applied as rehabilitation therapy and diagnosis mechanisms that emulate the movements performed by a patient with a therapist during the treatment, while the sensors placed on the exoskeleton measure the performance of the patient. This provides quantitative information about the recovery process of the patient, allowing the optimisation of the therapeutic treatment. For instance, a robotic device for the upper arm, based on impedance control, to guide patient's movements in specified trajectories has demonstrated the beneficial effects of the treatment of stroke patients (Krebs et al. 2002). There are also robots to assist or replace body functions in which the patient provides control signals to the device, while the exoskeleton provides most of the mechanical power required to perform the task. The human becomes a part of the system and feels a scaled-down version of the load that the device is carrying due to the force reflection (Kazerooni 1990). Finally, robots used to investigate biological motor control must be considered. However, in this type of robotic exoskeletons the main requirement is to apply and measure forces and positions precisely (Scott 1999).

One of the most important aspects in the field of wearable rehabilitation robots is the dual interaction between human and robot (Pons 2008). First, there is a cognitive interaction that allows the human to control the robot, while the robot provides some feedback to the human. In addition, the cognitive interaction results in a mutual adaptation in which robot and human effectively cooperate. Second, there is a physical interaction related to the application of controlled forces between both actors (Kazerooni 1990). The main concern of this physical interface is the safety, and other requirements are related to robustness and dependability of the robotic mechanism, taking into account the

${ }^{*}$ Corresponding author. Email: moreno@iai.csic.es 
characteristics of the human neuromuscular-skeletal system. For instance, a common drawback in robotic exoskeletons is that, in many cases, they are not suitable for wearable applications because of size and power-consumption restrictions, which become more critical when applied to a weak person suffering a disability.

Lower-limb orthoses are a widespread traditional solution to compensate lower-limb disorders, such as the muscular weakness affecting knee and ankle. Most of the solutions are purely passive, nevertheless, several researchers have proposed a number of devices as means to control the lower-limb joints actively. Myoelectric proportional control of pneumatic actuators has been proposed in Ferris et al. (2006), to artificially generate dorsal and plantar flexion torques in the ankle. These torques were proportional to the amplitude of the electromiographic signals measured in the soleus muscles. Variable-impedance control of ankle-foot orthosis to assist drop-foot gait was proposed by Blaya and Herr (2004). They discussed the application of a state machine with selective gait phasedependent control of a spring stiffness by means of a DC motor. In Kazerooni et al. (2006), the application of proportional control of position for the Bleex system was presented, a powered lower-extremity exoskeleton for human strength augmentation during locomotion. A spring-clutch design for a swing-phase knee orthosis controlled by a solenoid was presented by Irby et al. (1999). They proposed gait event detection based on the foot contact information, provided by force-sensitive resistors (FSRs). These events were used to release and lock the knee joint during gait. Gharooni et al. (2000) presented an orthosis with a controllable brake with the aim of improving the performance of functional electrical stimulation.

The application of intermittent control of resistance at joint level with a controllable exoskeleton, as a biomimetic joint impedance control, aims at being a clinically feasible solution. It solves the main drawbacks of energy consumption and weight. The analysis of the implementation of this approach in terms of the resulting motion of a post-polio patient with lower-limb weakness was presented in Moreno et al. (2008).

This paper presents an analysis of the interactions between the human user and the exoskeleton, following two phases of experimental procedures. This analysis is applied to this particular wearable lower-leg robotic exoskeleton. A description of the exoskeleton is presented along with the design requirements imposed by the human-device interactions, both physical and cognitive. The first phase of experimental procedures was designed to evaluate the interactions during learning with healthy subjects. Aditionally, with trained subjects, prolonged endurance tests were performed while walking on a treadmill as a requirement to move to the second phase. The second phase of experiments evaluates the interaction with users suffering lowerlimb weakness due to a post-polio syndrome. These re- sults illustrate the procedure to evaluate the performance of this lower-limb exoskeleton. The relevance of the methods to analyse human interaction with the exoskeleton is supported by the demonstration of the testing phases, covering the analysis of learning (training with healthy) to the analysis of the effects on real users (post-polio patient case).

\section{Methods}

A major challenge in the field of robotic exoskeletons is that the human being is placed at the centre of mechatronic technologies, and they have to interact with the human, leading to a mutual adaptation and functional enhancement. This led to the emergence of a new approach to systems development that has to take into account the interaction with biological systems (Pons 2008). In this section, the salient characteristics of this biomechatronic approach are discussed before focusing on the description of the lower-limb biomechatronic exoskeleton that will serve to illustrate the application of this design methodology. The mutual interaction between a mechatronic device and a biological system is twofold; it consists of a cognitive interaction and a physical interaction. The cognitive interaction between the exoskeleton and the human establishes a framework that enables (1) an effective control of the exoskeleton by the human and (2) the feedback of sensory and motor information back to the human.

The effective control of the exoskeleton by the human was based on a set of biomechanical and bioelectrical information provided by a collection of sensors included in the mechatronic exoskeleton. By means of this information and the corresponding data-processing algorithms, the exoskeleton could 'interpret' the human's intentions so that the combined biomechatronic system could be controlled. This cognitive interaction will be discussed in detail with respect to the detection of gait phases by a wearable lowerlimb exoskeleton through the interpretation of motion.

The goal of the physical interaction of a lower-limb exoskeleton is to provide walking stability to patients suffering from muscle weakness. In this case, patient users typically are not able to stabilise their knee joint (due to weakness), and the exoskeleton applies stabilising moments around the knee at the proper instants during the gait phases. The methodology proposed to analyse both the cognitive and the physical interaction in the design process is presented in the following sections with a case study: a wearable lower-limb exoskeleton. Regarding these procedures, it is important to highlight that it is expected that both cognitive and physical interactions will result in a mutual adaptation. In general, this implies that the human will modify the motor behaviour to adapt to the new status in which the exoskeleton provides compensation. Our approach analysed critical aspects in the design that affect the interaction, considering the human (in general biological structures) as a model in the design of technology. From a physical point of view the functional 


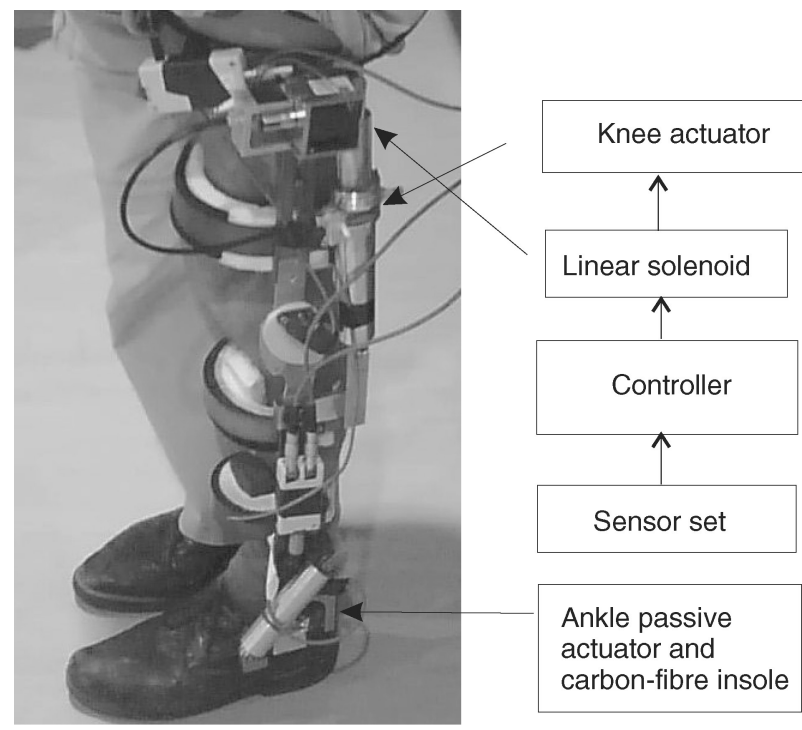

Figure 1. GAIT lower-limb exoskeleton, designed for functional compensation of gait.

movement anatomy, the dynamic behaviour of the musculoskeletal system and the human's motor structure were considered as models in the design of the exoskeleton's kinematics, actuation and control systems. From the cognitive point of view, the mutual adaptation mechanisms and learning behaviour have been considered.

\subsection{Lower-limb exoskeleton for functional compensation of gait}

\subsubsection{Design of the GAIT exoskeleton}

A wearable unilateral lower-limb exoskeleton was developed to enable functional compensation of people suffering muscle weakness around the knee joint 1 (Moreno et al. 2005).

The lower-limb exoskeleton followed the kinematic structure of the leg and spanned the knee and ankle joints. It consisted of segments for the upper and lower legs as well as a carbon-fibre insole for the foot.

The compensation of the GAIT exoskeleton corresponded to an impedance modulation during gait. The low musculoskeletal impedance of the user was modified by the exoskeleton so that the combined biomechatronic system (including both actors) resulted in a more physiological gait.

The GAIT exoskeleton was equipped with a knee actuator that mechanically mimics the behaviour of the human knee during gait (see Figure 1). This was done by switching either mechanically or electronically between two springs contained in the knee actuator. These springs were aimed at reproducing the mechanical behaviour of the knee musculoskeletal system during the stance (ST) and swing (SW) phases. The required torque to stabilise the knee for a 100- $\mathrm{kg}$ patient during ST was of the order of $70 \mathrm{Nm}$. This high torque must be provided at the gait rate which demands high power and energy. Current actuator technologies and power supplies capable of providing these driving characteristics are too bulky and heavy for an ambulatory exoskeleton. In addition, the high power consumption could limit the use of the exoskeleton to a few walking cycles. The knee-joint stiffness during ST and gait was taken as a basis for the design of the actuator system in the GAIT exoskeleton. A rigid spring was defined to be applied during ST, in order to absorb body weight and partially assist push-off to start the SW phase. A soft spring was used to allow knee flexion during SW and provide support to the knee extension to prepare for foot contact. This is illustrated in Figure 2 that plots the knee-joint torque versus the knee angle for a healthy person. It clearly shows different areas of behaviour, depending on whether the knee provides support to the body weight during ST with high stiffness or in a low-stiffness condition during the SW. The stiffness of each spring was defined according to the body weight of the subject, with the aim to approach a normal profile. A detailed description of this design can be found in Moreno et al. (2005).

The transition between both states could be triggered either mechanically or electronically. In the case of pure mechanical activation, a cable-driven (called here CD) mode was actuated during ankle dorsiflexion. This mechanism switched from the rigid to the soft spring during the ankle dorsiflexion that occurs before toe-off. The degree of ankle dorsiflexion required to provide action was adjustable.

The electronically driven mode of control (called here CC), by means of the linear solenoid activated at the level of the knee, was based on the kinematics of the leg and the foot captured by inertial sensors. The knee actuator was batterypowered $(12 \mathrm{Vdc}, 1.2 \mathrm{Ah})$ to provide solenoid actuation. For a 500-ms duty cycle and a permanent rate of discharge of $0.5 \mathrm{~A}$, the estimated discharge time is $2.5 \mathrm{~h}$.

At the ankle joint, a passive actuator enabled a control spring for plantar flexion during SW, and a second spring compressed in dorsiflexion. An in-shoe carbon-fibre insole was included to assist the push-off by an energy storagerelease principle.

The kinematic sensors were aimed at measuring the ankle and knee joint angles in the sagittal plane. Two inertial sensor blocks (Inertial measurement units IMUs) were placed in the foot and shank, in order to measure the ankle angle. Each IMU contained one gyroscope, which measures the angular velocity in the sagittal plane, and two linear accelerometers oriented in the vertical and anteroposterior axes, which measure the linear acceleration. The knee angle was measured with an angular potentiometer. The kinetic sensors measured the external forces between the foot and the floor. Two (FSRs) were placed on the exoskeleton foot sole at the zones of the heel and the forefoot. 


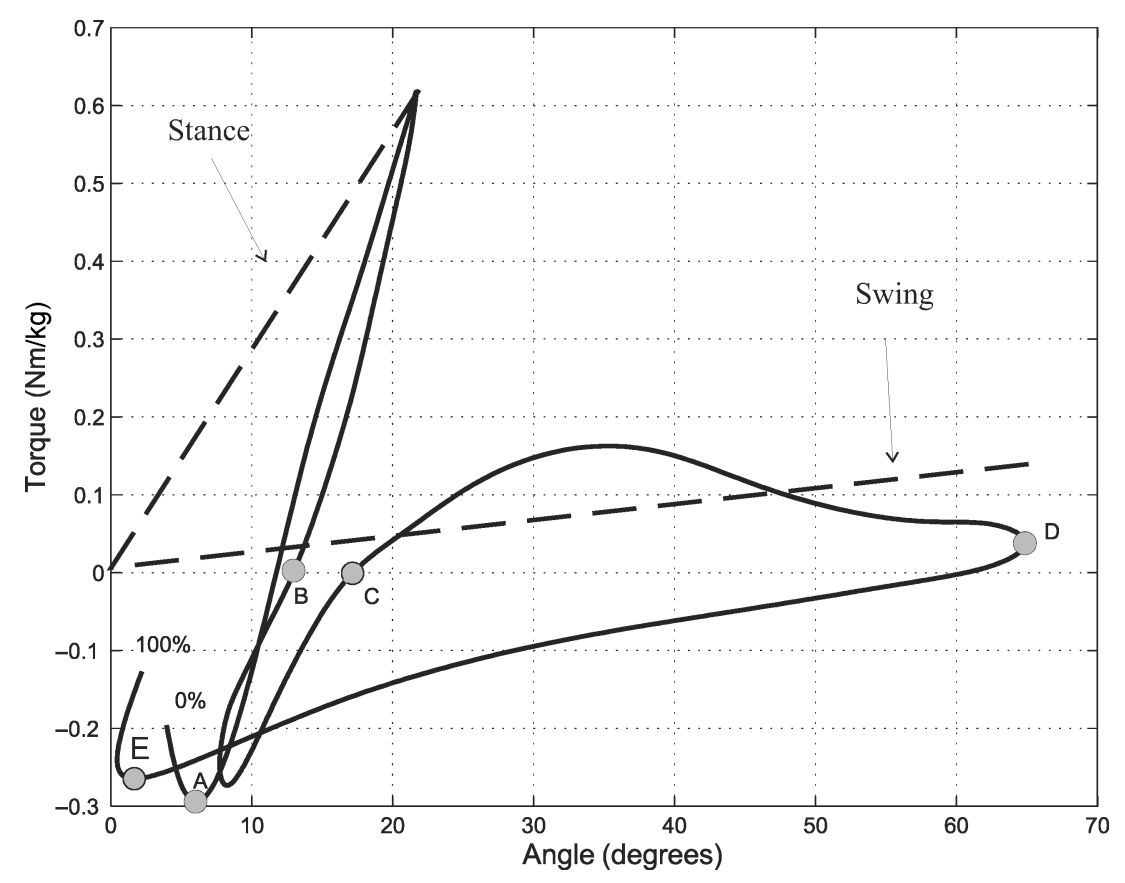

Figure 2. Model of the knee musculoskeletal system during gait represented as the torque (normalised to body weight) and the joint angle. The dashed lines represent the approximations by ideal springs. The solid lines represent the actual behaviour of the knee obtained from average measurements (Winter 1991). The dashed line marked 'Stance' approximates the stiffness during the ST phase (points A to B). The dashed line referred to as 'Swing' represents an approximation of the stiffness during the SW phase (points D to E).

The weight of the complete GAIT exoskeleton was $3 \mathrm{~kg}$. The weight of the ambulatory unit alone was 370 . An important aspect of an exoskeleton for the compensation of the leg weakness is safety. In this case, the exoskeleton must prevent the knee from collapsing during the ST phase of gait, which would lead to a fall of the patient. Therefore, the subject must be able to control the knee mechanism under any circumstance. The safety strategy defined was reactive for those patients at risk of falling (Moreno et al. 2005).

The behaviour of the GAIT exoskeleton is sketched in Figure 3. During the ST phase, the knee actuator applied a high-stiffness spring - K1 - during a period of time to provide joint stability. During the SW phase, the actuator applied a low-stiffness spring - K2 - to store and recover spring energy, assisting knee extension prior heel contact. The ankle was controlled passively by means of two compensation springs: K3 and K4. The knee joint can be released when the ground reaction force (GRF) vector is ahead of the knee-hinge joint in the antero-posterior direction. In this way the GRF creates an extension moment around the knee, thus locking the knee in extension.

\subsubsection{Cognitive interaction: reactive control based on gait-phase detection}

The GAIT exoskeleton had to identify the different phases of the gait cycle and control the shift between SW and ST actuators. In order to provide safe transition from ST to $\mathrm{SW}, \mathrm{CD}$ mode required the adjustment of the degree of ankle dorsiflexion. In the case of $\mathrm{CC}$ mode, it was required the definition of tresholds applied to inertial-sensing signals. Transition from SW to ST was achieved mechanically when the knee recovered full extension, both in $\mathrm{CD}$ and CC modes. CD mode was designed for the first phase of evaluation of training with healthy subjects. CC mode was intended for the second phase of evaluation with a patient case.

An example of representative graphs of the combined human-exoskeleton kinematics in the sagittal plane can be seen in Figure 4. The leg deceleration and landing could be distinguished in the foot-bar longitudinal acceleration (y-axis) signal. The zero acceleration of the foot bar during stance and the sign of linear rotation measured by the accelerometer featured a correlated pattern with shank acceleration component during ST and SW progressions. Ankle-joint velocity could be estimated and joint angle was calculated by cycle-to-cycle integration. Based on zerovelocity detection by the inertial set, drift could be removed periodically. A rule-based algorithm could be implemented to identify particular gait events under CC mode. The algorithm allowed the detection of the transition instant (TI; ST detection) between ST and SW phases during gait. This instant detection was used to switch between the two actuators modelling the musculoskeletal system in ST and SW. 


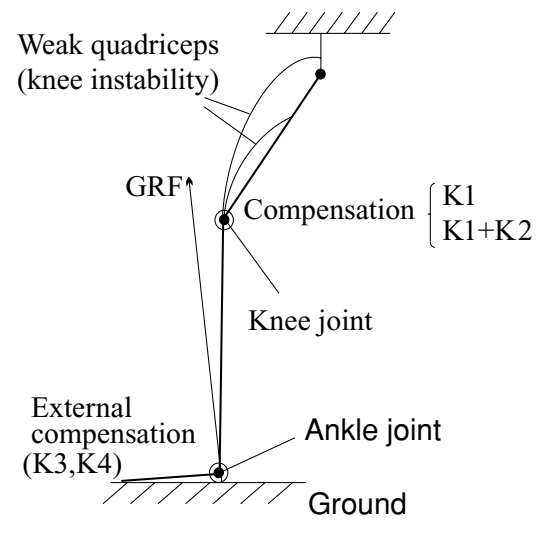

(a)

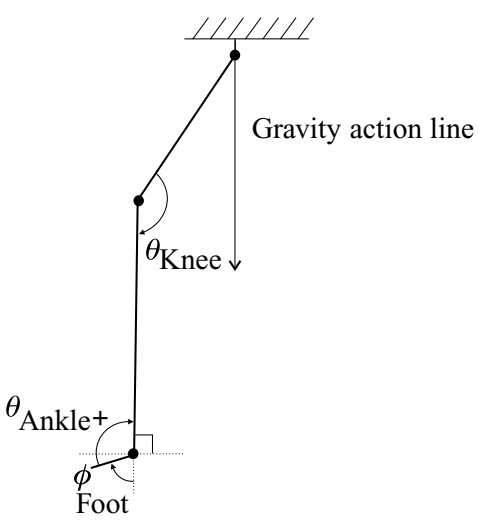

(b)

Figure 3. Exoskeleton functional scheme: (a) scheme of the weak (quadriceps) lower leg with the exoskeleton; (b) Relative joints angles in the system.

The partial-control strategy objectives of the knee in gait cycle were:

(1) heel contact with footfall control, followed by stabilised knee extension through $\mathrm{K} 1$ at terminal SW;

(2) heel off during ST phase after controlled flexion of the knee;
(3) during pre-SW rate of turn of the shank sign change and knee release by $\mathrm{K} 1$ to $\mathrm{K} 2$ transition;

(4) shank rotation during knee flexion and assistance provided by $\mathrm{K} 2$ - partially charged by inertia - to extension at the end of cycle.

The TI of this intermittent mechanism that controls the knee actuator could occur at any kinematic configuration

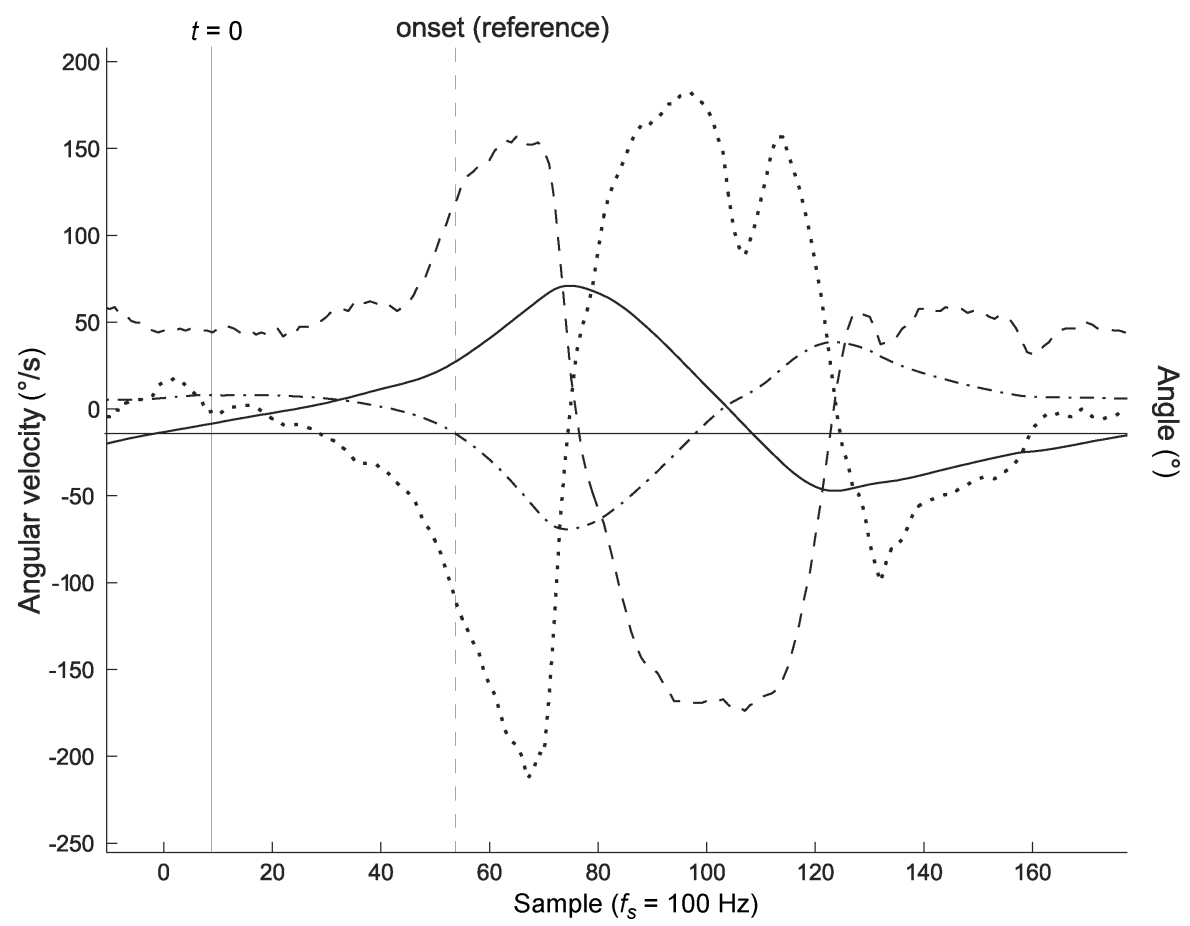

Figure 4. Lower-limb exoskeleton kinematic data used to establish the cognitive interaction framework: typical pattern of foot (continuous) and shank (dashed-dotted) segments rotations and foot (dashed) and shank (dotted) rates of turn, in sagittal plane, during a walking task at $34 \mathrm{~m} / \mathrm{min}$ speed (CD mode, after training of a healthy subject). A system with a cable triggers the knee mechanism, depending on a fixed degree of dorsiflexion. 
of the leg. The goal of the controller under CC mode was to detect the TI with the information, sampled at $100 \mathrm{~Hz}$, from the IMUs. In order to define the set of rules for the discrete controller of the knee for gait compensation, the features of the input signals were analysed at different gait speeds (low, medium, high) and different step lengths with an experimental data set. The binary state of the knee actuator was monitored with a pressure-sensitive resistor (thickness $=0.3 \mathrm{~mm}$ ) placed in the knee-unlocking mechanism. This binary information was used as a reference to determine experimentally the TI and the signal amplitudes as functions of the gait velocity. For the foot and shank segments, it was valid to calculate the rate of turn of each segment, between mid-ST phase and TI during pre-SW, along the axis of rotation in the sagittal plane. The condition for the detection of the TI in the controller was based on the rates of turn, applying upper and lower thresholds to the angular velocities of the foot and shank, as described in Moreno et al. (2008).

The algorithm under CC (see Figure 5) evaluated the angular velocities of the leg and foot segments in the margins given by the upper and lower thresholds. As the foot was in contact with the ground, the rotation of the foot was related to the ankle dorsiflexion. The threshold of the vertical acceleration of the foot was evaluated when the foot was lifted off the ground. During the SW phase, the algorithm evaluated the system state and the level of knee flexion to guarantee the avoidance of unsafe activation of the linear solenoid in the last portion of the SW that could compromise the safety of the patient. The output of the TI detector in each cycle was a square pulse with variable width and a rising edge time $\delta T$. This pulse activated the linear solenoid that originated the transition to SW in the intermittent mechanism. The pulse width should be enough to free the knee for a sufficient time and allow the knee flexion at early SW. The maximal pulse width was limited by the half of the SW duration.

\subsection{Experiments with subjects}

\subsubsection{Phase 1: learning experiments with healthy subjects}

The learning and use of the new lower-leg exoskeleton, under CD mode, was investigated experimentally with four healthy subjects (male, mean age $23 \mathrm{yr}$ ). An exoskeleton incorporating customised linear springs, one for support (K1) and one for SW (K2) at the knee joints, was adapted to each participant $(\mathrm{N}=5)$. The transition between each spring in the knee actuator was determined by the ankle dorsiflexion at toe-off and by default was set by default at $15^{\circ}$. There were four experimental conditions: (1) normal gait without exoskeleton, (2) gait with orthosis without actuation, (3) gait with actuated orthosis but without learning and (4) gait with orthosis after 30-min learning. The learning periods consisted of walking and a series of dorsiflexion movements inducing the transition between the springs. Tuning of the angle of dorsiflexion required to control the actuator was performed progressively along the gait trials, taking into account the opinion of the user and the observation of gait profiles. The trials were classified as successful or not if the subject managed to induce transition to K1 before ST and to unlock it at the end of ST.

The participants had to adapt their gait pattern until they learnt how to control the GAIT exoskeleton. The experiments were divided in four blocks with five valid trials: (1) gait with orthosis before any training, (2) gait with orthosis after 15-min training, (3) gait with orthosis after another

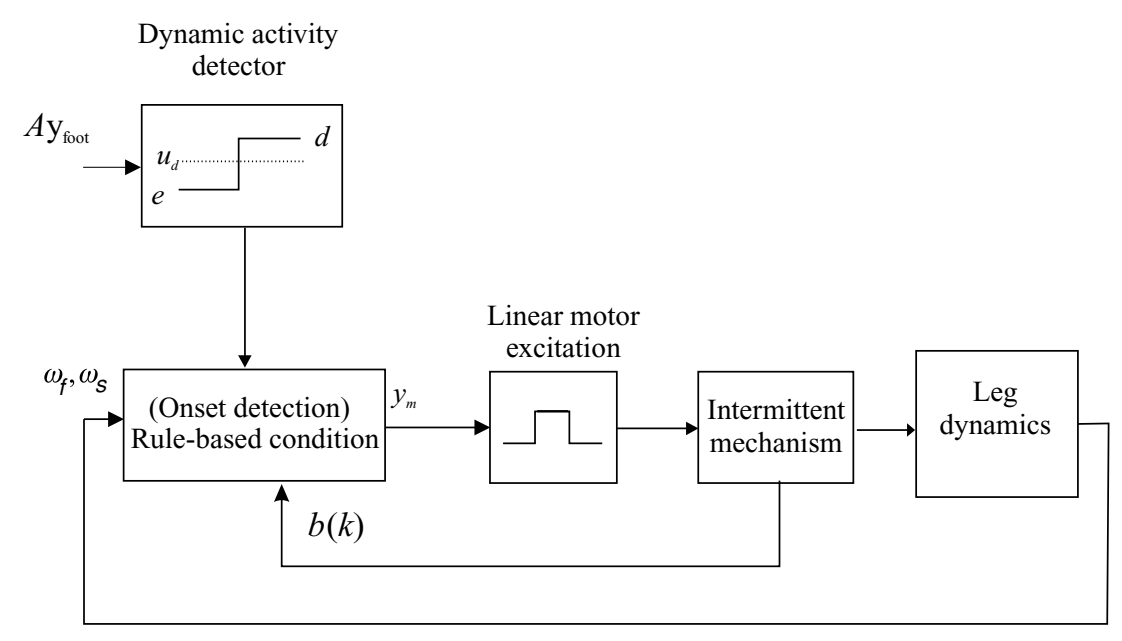

Figure 5. The rule-based block, based on motion information, generated signals to trigger the knee actuator. The status of the knee $b$ was tracked for the decision making. 
15-min training and (4) normal gait without orthosis. The lower-limb motion was recorded with an optical measuring device.

The lower-limb motion, electromyography (EMG) data and GRFs were measured in each trial. The EMG electrodes were placed bilaterally at the gastrocnemius, tibialis anterior, biceps and rectus femoris, following the SENIAM recommendations (Freriks and Hermens 1999). The raw EMG data was rectified and filtered (low-pass $6 \mathrm{~Hz}$ ) to obtain the EMG gait patterns in each condition.

A dedicated test has been performed to test the safety of GAIT system, with three healthy subjects $(75-80 \mathrm{~kg})$ who participated in the weight-bearing experiment. During this experiment the healthy subjects were asked to put as much as weight in the orthosis during a number of seconds. Observation of the fact that the GAIT orthosis sustains the tests were regarded as a good indication that the system was indeed capable in sustaining the weight of healthy subjects in the tested range of body weight. Thus, a 1000-step endurance test was performed by the three healthy subjects. During the test the subjects were asked to walk at a treadmill at a constant speed and asked to count the number of steps in which according to his/her opinion the knee lock/unlock mechanism did not function properly. The ambulatory unit recorded the knee angle during the trials, as well as whether the knee joint was locked and unlocked.

Aditionally, an analysis of gait variability was performed in order to evaluate the effects of stride-to-velocity fluctuations. These experiments were performed with one healthy subject at different speeds and step lengths. The trials consisted of walking back and forth along a $10-\mathrm{m}$ path, each trial setting the step length with marks on the floor and defining the gait speed by means of a metronome, with systematic adjustments of the cable mechanism to provide a comfortable gait pattern (see Table 1). The gait velocity and step length variations were defined according to average values taken from Perry (1999), considering realistic combinations of $100 \%, 70 \%, 60 \%$ and $50 \%$. Some combinations were not feasible (high stride length and low step frequency). The results provide a characterisation of the spatio-temporal parameters that can be used for online adjustment of the decision-making algorithm, as presented in Moreno et al. (2008).

\subsubsection{Phase 2: experiments with post-polio patient}

In order to asses the immediate effects of the knee-anklefoot orthosis (KAFO), one patient with post-polio syndrome with demand of a technical assistance for walking and an active lifestyle has been involved. The tests were conducted with protocol approval given by the Instituto de Biomecánica de Valencia-UPV (IBV) medical ethical committee.

The participant was one male (body weight $76 \mathrm{~kg}$; height $172 \mathrm{~cm}$; age 46 years) affected by poliomyelitis during childhood, with bilateral loss of muscle power and more impact on the left leg. The subject declared having rejected the use of prescribed KAFOs in the past and a preference for the daily use of crutches. A muscular balance test was carried out following the muscle manual test (MMT) of the medical research council (IBV). Poliomyelitis affected with primary weakness the left (second degree) and right (second degree) ankle dorsiflexors, the left isquio-tibilalis (first degree) and the left quadriceps group (first degree).

The unilateral KAFO was adapted to the subject. The construction of the hinges restricted motion to the sagittal plane. During each gait trial with $\mathrm{CD}$, progressive adjustment of the degree of dorsiflexion, providing SW mode of the knee, was performed at a self-preferred speed. With a satisfactory configuration, five walking trials (assisted by crutches) through a $10-\mathrm{m}$ path were performed. The kinematic data measured with $\mathrm{CD}$ mode was used to define the parameters for the CC mode. During static condition with full body weight unloading, the pulling action capability of the solenoid was tested in order to proceed with the walking trials at self-preferred speed. If an increase in confidence on the weight support provided by the exoskeleton was revealed, gait without crutches was tested to measure GRFs.

The GRFs of the leg were measured with a dynamometric platform (active area $600 \times 370 \mathrm{~mm}$, range 2000 $15000 \mathrm{~N}$ in vertical force, Dinascan/IBV), when walking without external aids was feasible after training.

The components of the GRFs were normalised to ST-phase percentage of the duration. The reaction force data was resampled with averaging purposes and comparison between conditions. From the dynamometric platform measurements, average values of braking propulsion and

Table 1. Systematic variations of normal subject walking with the CD prototype.

\begin{tabular}{lcccccc}
\hline & $\begin{array}{c}\text { Step } \\
\text { Percentage } \\
\text { length }(\mathrm{m})\end{array}$ & $\begin{array}{c}\text { Stride } \\
\text { length }(\mathrm{m})\end{array}$ & \multicolumn{3}{c}{ Speed (m/min) } \\
\hline 100 & 0.73 & 1.46 & 81.0 & 56.7 & 48.6 & 40.5 \\
70 & 0.51 & 1.02 & 56.7 & 39.7 & 34.0 & 28.4 \\
60 & 0.44 & 0.88 & 48.6 & 34.0 & 29.2 & 24.3 \\
50 & 0.37 & 0.73 & 40.5 & 28.4 & 24.3 & 20.3 \\
& & Cadence (step/min) & 111 & 78 & 67 & 56 \\
& & Metronome (bpm) & 1.85 & 1.30 & 1.11 & 0.93 \\
\hline
\end{tabular}


push-off forces were calculated by means of the NEDAMH/IBV with Kinescan/IBV measurements. Normative data of the three components of the GRFs in normal gait was considered as reference (database provided by IBV). In order to compare the results in terms of amplitude with the reference data, the root mean square differences (RMS) were utilised. Coefficient of multiple correlation (CMC) was calculated to evaluate the similarity between the reference and the force data.

\section{Results}

The result of the (cognitive and physical) interaction between human and exoskeleton was a mutual adaptation and cooperation. This was evident in the changes of motor patterns during this interaction by analysing how the human gait patterns were modified after interaction with the unilateral lower-limb exoskeleton.

\subsection{Phase 1: learning tests}

In some trials the orthosis was not switched from the rigid spring (K1) to the flexing knee (K2), and they were marked as unsuccessful trials. However, the reverse, knee flexing during weight loading in ST, which is potentially dangerous for the patient, never occurred. There was an improvement in the use of the exoskeleton because the number of unsuccessful trials decreased and vanished after a training period of $2 \mathrm{~h}$ (see results in Table 2). The joint angles in the sagittal plane showed remarkable differences before and after the training. Specially, the knee-joint angles of the leg with exoskeleton are more similar to the normal gait values, as can be seen in Figure 6.

Regarding the EMG patterns during the learning tests, the experimental data suggested that subjects need to 'learn' to use the orthosis. The duration of the left and right steps was slightly increased during the learning process, and there was a large variability in the step durations (see Figure 7). The changes in the gait patterns were reflected in the EMG patterns of activity. During the learning process the EMG activities were less stereotyped, and the averaging of these trials resulted in large standard deviations. The bursts were also larger than normal and had longer durations.

It was remarkable to see the modification of the timing and amplitude of the gastrocnemius of the left leg fitted

Table 2. Number of trials in which the exoskeleton knee actuator switched successfully.

\begin{tabular}{lccc}
\hline & $\begin{array}{c}\text { No } \\
\text { training }\end{array}$ & $\begin{array}{c}\text { First training } \\
\text { series }\end{array}$ & $\begin{array}{c}\text { Second training } \\
\text { series }\end{array}$ \\
\hline Unsuccessful & 4 & 2 & 0 \\
Successful & 3 & 5 & 5 \\
Total & 7 & 7 & 5 \\
\hline
\end{tabular}

with the orthosis during the experimental session. With respect to the endurance tests, in the cable-operated system the error rate was $2.4 \%$ based on the experimental validation that consisted of 1000 consecutive strides measured on a treadmill. From the data and the subjective counting with the cable-operated system it was concluded that for the three subjects the mechanism featured error rates of $1.9 \%, 2.9 \%$ and $2.4 \%$ out of 1000 steps. However, these error rates were significantly reduced under CC mode that reached a success rate of $99 \%$ in the tests at low and medium velocities.

\subsection{Phase 2: patient case}

An analysis of the influence of the exoskeleton as compared to the subject's pattern and normal profiles was performed. A reduction of medio-lateral reaction forces was distinguished from the calculated mean values. The need of lateral movement frequent in post-polio syndrome patient was reduced by this fact, as can be seen in Figure 8. The need of lateral movement was due to the leg abduction during SW with the affected leg.

The profile obtained of vertical GRFs with the CC approximates to the normal profile with a CMC above 0.94 . In this comparison, the RMS errors were less than $6 \%$ of the full range of the measurements.

An increase in the magnitude of the push-off force with $\mathrm{CC}$ was found, when compared with the CD system. From the calculated average forces during braking, propulsion, and push-off (Table 3) no significative differences were found for shock absorption, and surprisingly, maximum mean value of the vertical GRFs during the propulsion was higher with $\mathrm{CD}$, a fact that could also be found in the difference between maximum values of plantar flexion during SW.

After a period of time, the subject was able to walk with a free-swinging leg (average peak flexion of $50^{\circ}$ ) with the two crutches (mean peak values of maximum flexion joint angles in Table 4). With the final configuration of the exoskeleton, the subject succeeded to walk with a free knee during the SW phase. The self-selected speed was low, below $4 \mathrm{~km} / \mathrm{hr}$, and the free-swinging leg revealed average peak flexion of $50^{\circ}$ and a slight controlled flexion during ST. The plantar fall was limited by the actuator system at maximum $5^{\circ}$.

\section{Discussion}

In this paper the human interaction with a wearable exoskeleton has been analysed from different perspectives.

The results of the analysis with healthy subjects during learning to use the exoskeleton demonstrated how the human user adapts by applying slight modifications of the gait pattern to ensure proper functioning of the exoskeleton. This implied that at the beggining of the process of tuning the exoskeleton, it was mandatory to 

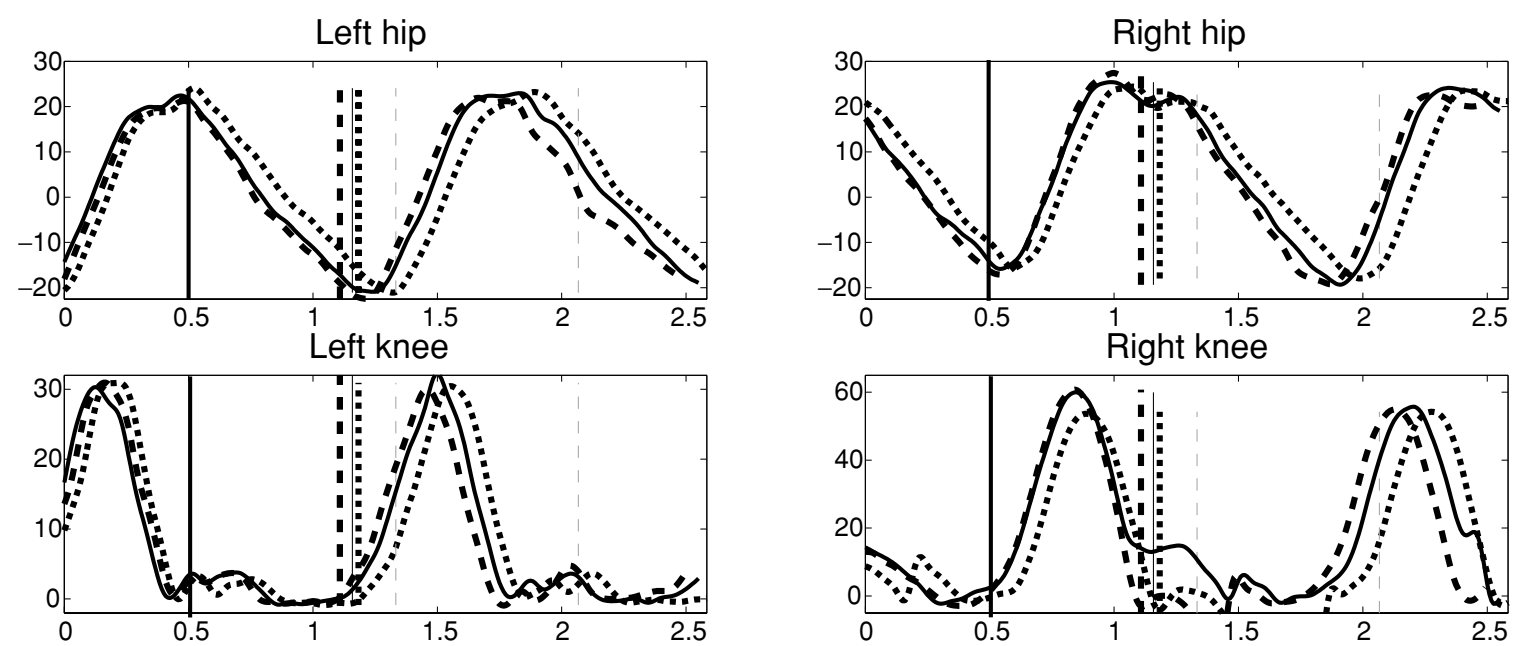

(a)
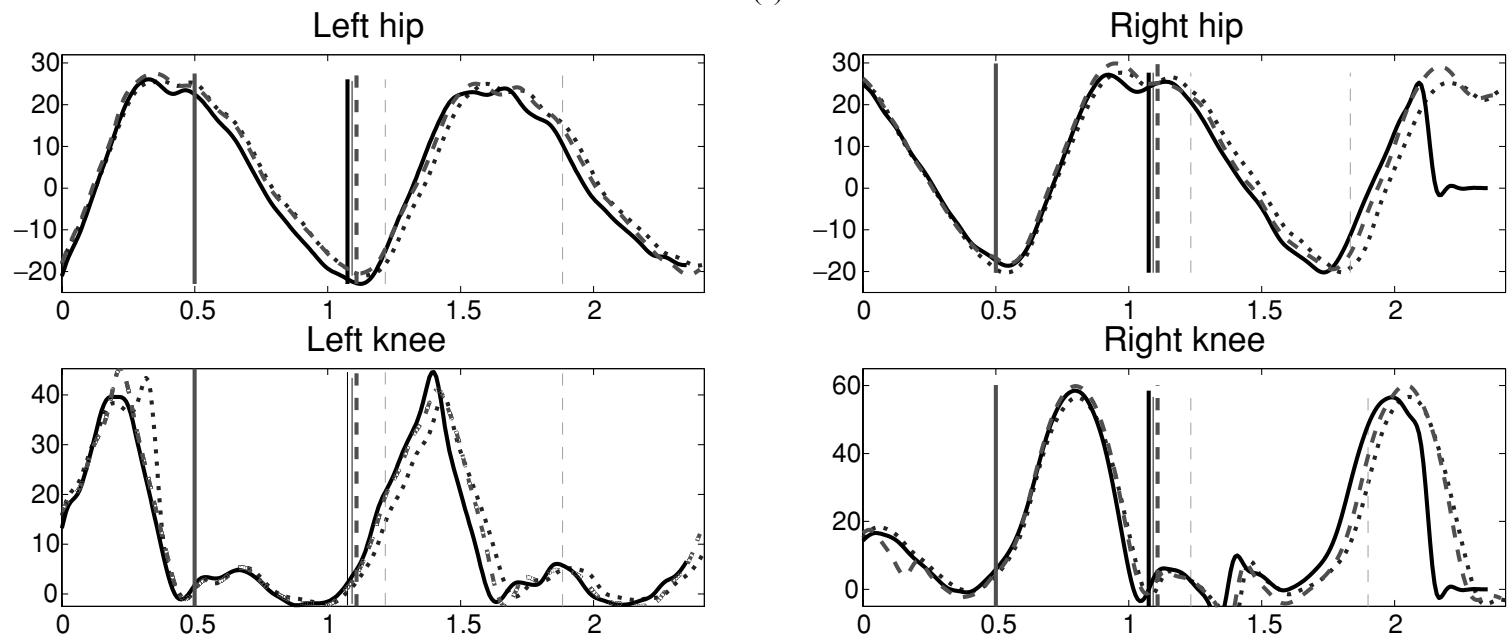

(b)

Figure 6. The hip- and knee-joint angles (degrees) versus time (s) during three trials (solid, dashed and dotted lines) measured (a) before training and (b) after training. All the trials are aligned to the left-heel strike at $0.5 \mathrm{~s}$ (vertical solid line) of the 2.5 -s time window. The other vertical lines (solid, dashed and dotted) indicate the right-foot contacts.

perform a step-wise tuning procedure of the control parameters during training under controlled conditions. The tuning could be done with iterative definition of the parameters with the goal of minimising the occurrences of a locked knee during the SW phase. In the medium-term application (daily conditions), more complex modifications are expected, which are related to gait speed and stride length.
The changes in the timing and amplitude of the gastrocnemius of the left leg fitted with the orthosis revealed gait adaptation mechanisms, as found in previous studies (Forner-Cordero et al. 2006). The burst of activity of this muscle that had to provide a plantar-flexion torque was delayed with respect to normal gait. It is suggested that this occurred in order to guarantee a sufficient dorsiflexion to unblock the knee. The peak of activity was larger because

Table 3. Mean values of kinetics for the orthotic leg during the ST phase of gait (low cadence) for the testing conditions.

\begin{tabular}{lccccrr}
\hline Condition & $\begin{array}{c}\text { ST period } \\
(\mathrm{s})\end{array}$ & $\begin{array}{c}\text { Velocity } \\
(\mathrm{m} / \mathrm{s})\end{array}$ & $\begin{array}{c}\text { Braking } \\
(\mathrm{N})\end{array}$ & $\begin{array}{c}\text { Propulsion } \\
(\mathrm{N})\end{array}$ & $\begin{array}{c}\text { Push-off } \\
(\mathrm{N})\end{array}$ & $\begin{array}{c}\text { Weight } \\
(\mathrm{Kg})\end{array}$ \\
\hline Exoskeleton CC & 0.84 & 0.93 & 62.57 & 81.37 & 769.24 & 72.71 \\
Exoskeleton CD & 0.83 & 0.99 & 62.21 & 88.96 & 757.48 & 70.92 \\
\hline
\end{tabular}




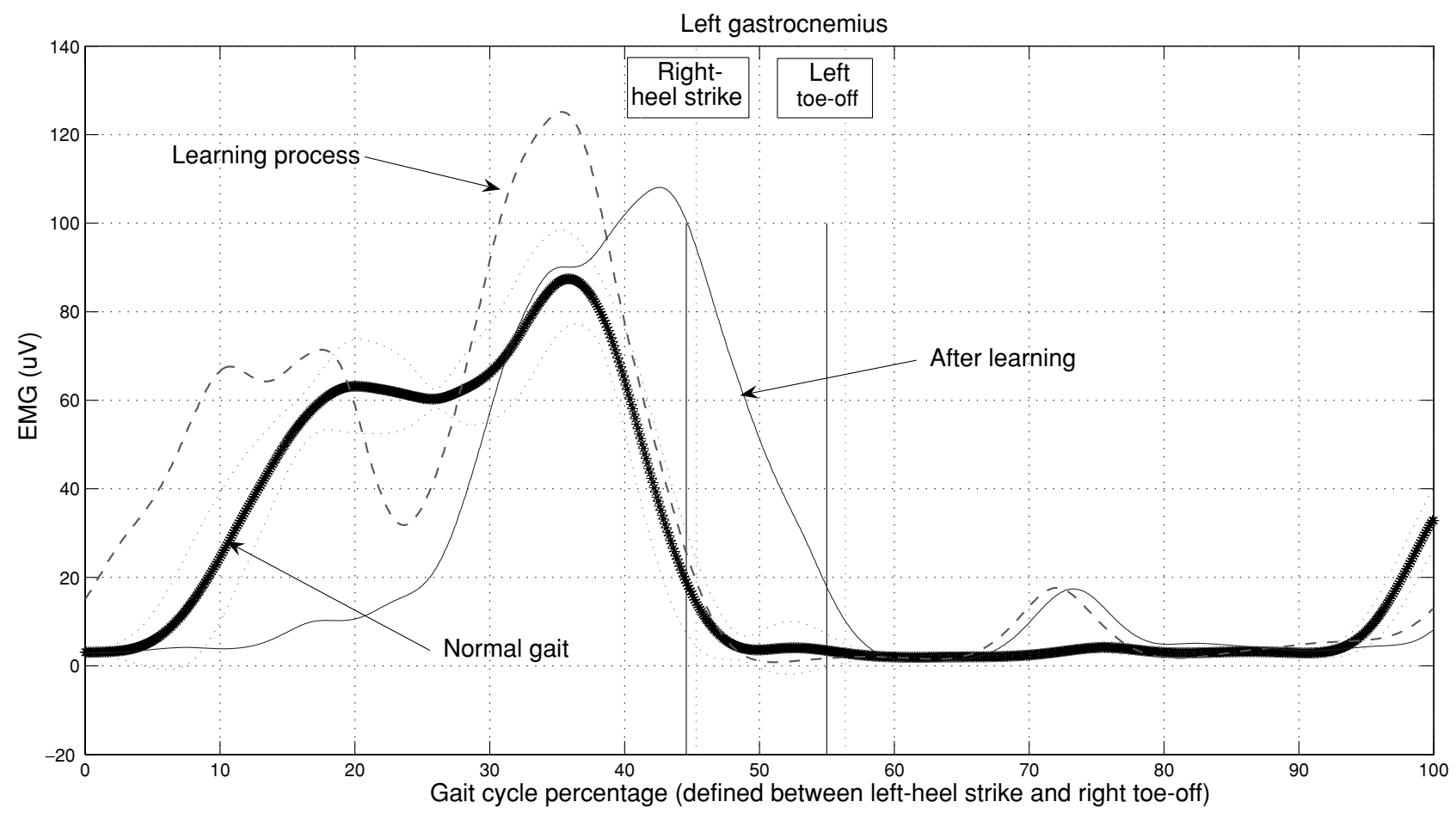

Figure 7. EMG gait patterns of the left gastrocnemius:t normal (thick), with orthosis before (dashed) and after (solid) learning.
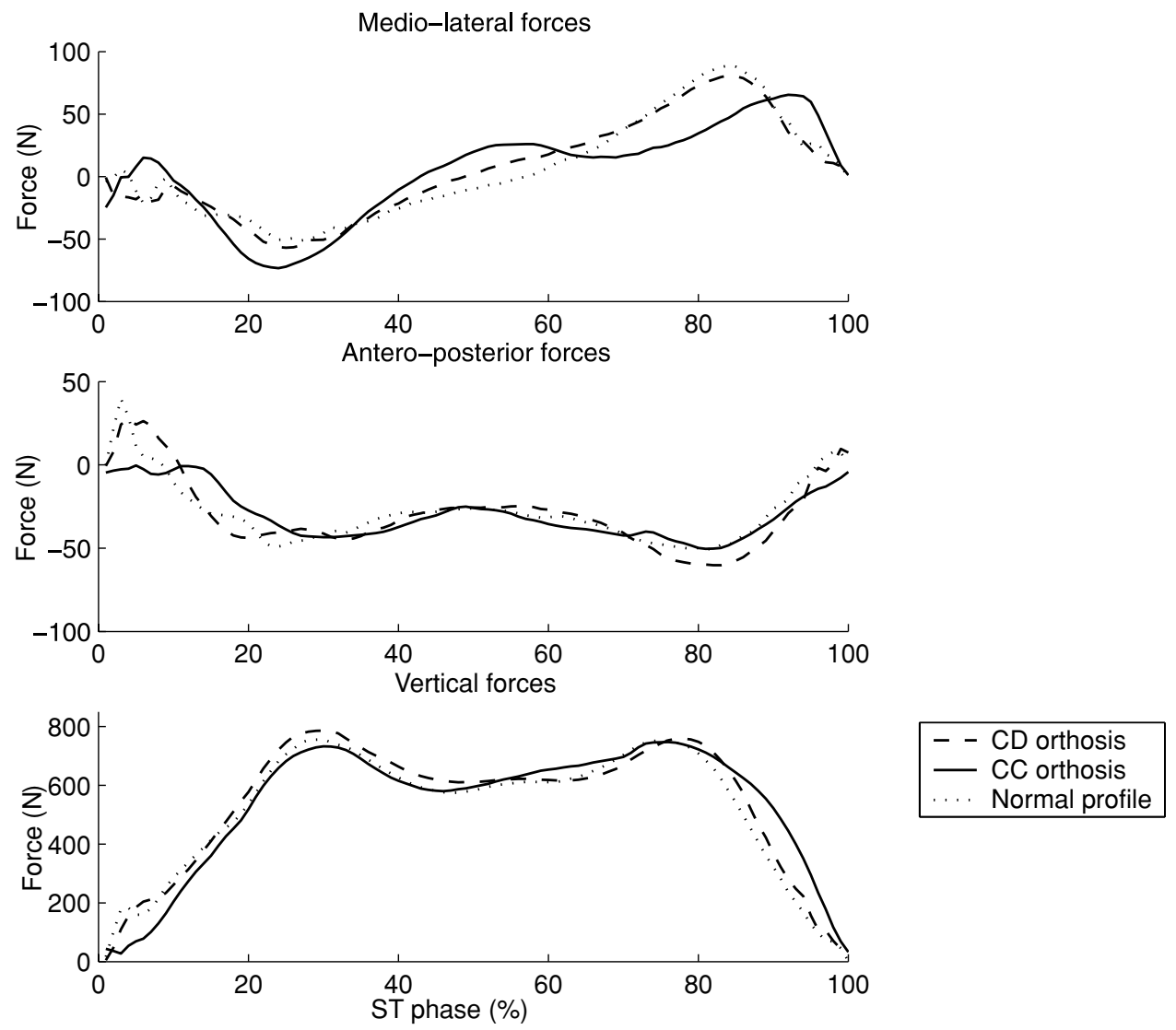

Figure 8. GRFs (mean values): evaluation data of patient under the testing conditions and average normal profile of walking with the exoskeleton. 
Table 4. Mean peak values of maximum flexion joints angles during ST and SW phases of patient trials.

\begin{tabular}{lrr}
\hline & \multicolumn{1}{c}{$\mathrm{CD}$} & \multicolumn{1}{c}{$\mathrm{CC}$} \\
& $(5$ cycles $)$ & $(25$ cycles $)$ \\
\hline Knee flexion (ST) $\left[^{\circ}\right]$ & $5.5 \pm 2$ & $5.5 \pm 1$ \\
Knee flexion (SW) ${ }^{\circ}$ ] & $61 \pm 5$ & $61 \pm 3$ \\
Dorsiflexion (ST) ${ }^{\circ}{ }^{\circ}$ & $6 \pm 3$ & $5 \pm 2$ \\
Plantarflexion (SW) $\left[{ }^{\circ}\right]$ & $22 \pm 5$ & $20 \pm 3$ \\
\hline
\end{tabular}

it had to provide sufficient push-off momentum during a shorter amount of time. In addittion, a larger peak in the push-off is generated in order to provide sufficient energy to the leg at the beggining of its SW phase. This added energy should be enough to extend (lock) the knee at the end of the SW.

An open question left for further research is the analysis of gait variability (Forner-Cordero et al. 2006) and the incorporation of adaptative mechanisms that can cope with such variability, such as neural oscillators and modifications in the magnitudes of the inputs that command the decision-making defining, for instance, fuzzy rules.

The reported results with the patient supported the contention that the externally applied stiffness with the controllable exoskeleton provided sufficient support during ST phase in this patient case. The profile obtained of vertical GRFs with the stable ST phase approximated the magnitudes observed in healthy subjects. The knee-joint control under $\mathrm{CD}$ mode revealed the need by the patient to use his body (back and hip) to dorsiflex the foot and hence unlock the system. A more natural dorsiflexion trajectory at terminal ST could be obtained, controlling this exoskeleton with the feedback provided by the motion sensors. This fact can be associated to the higher level of push-off force if compared with the exoskeleton under CD mode. The higher peak in the vertical force agrees with results found in the case of healthy subjects. The findings of this study provide valuable information for the optimisation of the exoskeleton prototypes and improving their ease of control. In general, previous studies have been focused on providing a free SWphase motion while preventing the knee flexion during, ST (Kaufman et al. 1996; Irby et al. 1999). The reduction of mid-lateral forces seen in this study might imply the ability to acquire a much more physiological gait, preventing from the lateral movement, very common in this kind of patients. Currently, we are examining the situation during the SW phase and, in particular, the effects of the spring for extension assistance during the initiation of the oscillation and prior to heel contact, (Forner-Cordero et al. 2007).

There are two main aspects in the presented biomechatronic approach: It must be designed for the human, considering the interactions, which could be cognitive or physical. These interactions pose some requirements in the design that claim for technological solutions. During the inter- action an adaptation of the human actor was observed in the modification of gait patterns. In turn, the variation in the motor output modifies the exoskeleton behaviour. It is suggested that adaptive/learning capabilities must be fully implemented in next generation biomechatronic robotic exoskeletons.

\section{Acknowledgements}

The authors thank Ossur hf and IB Valencia for their contributions in materials and experimentation. We also acknowledge the enormous contributions and support from J.M. Baydal in testing and data analysis. The work presented in this paper has been partially founded through grants IST-2001-37751 (GAIT, intelligent knee and ankle orthosis for biomechanical evaluation and functional compensation of joint disorders) and IST-61-045301-STP (ESBIRRO, Biomimetic actuation, sensing and control technology for limit cycle bipedal walkers) of the European Commission.

\section{References}

Barnett S, Bagley A, Skinner H. 1993. Ankle weight effect on gait: orthotic implications. Orthopedics 1993(16):1127-1131.

Blaya J, Herr H. 2004. Adaptive control of a variable-impedance ankle-foot orthosis to assist drop foot gait. IEEE Trans Neural Syst Rehabil Eng. 12:24-31.

Carrozza MC, Massa B, Dario P, Zecca M, Micera S, Pastacaldi P. 2002. A two DoF finger for a biomechatronic artificial hand. Technol Health Care 10(2):77-89.

Ferris D, Gordon K, Sawicki G, Peethambaran A. 2006. An improved powered ankle foot orthosis using proportional myoelectric control. Gait Post. 23:425-428.

Forner-Cordero A. 2007. Reduction in reflex latencies of the rectus femoris during gait perturbation experiments. Mot Control 11(Suppl): S178.

Forner-Cordero A, van der Helm FCT, Koopman HFJM. 2006. Describing gait as a sequence of states. J Biomech. 39(5):948 957.

Freriks B, Hermens HJ. 1999. SENIAM 9: European rcommendations for surface electromiography, results of the SENIAM project [D-rom]. Roessingh Research and Development, Enschea.

Gharooni S, Heller B, Tokhi MO. 2000. A new hybrid spring brake orthosis for controlling hip and knee flexion in the swing phase. IEEE Trans Rehabil Eng. 9(1):106-107.

Harwin W, Leiber O, Austwick G, Dislis C. 2002. Clinical potential and design of programmable mechanical impendances for orthotic applications. Robotica (16):523-530.

Irby S, Kaufman K, Wirta R, Sutherland R. 1999. Optimization and application of a wrap-spring clutch to a dynamic kneeankle-foot orthosis. IEEE Trans on Rehabil Eng. 7(2):130 134.

Kaufman KR, Irby SE, Mathewson JW, Wirta RW, Sutherland DH. 1996. Energy-efficient knee-ankle foot orthosis: a case study. J Prosthet Orthot. 8(3):79-85.

Kazerooni H. 1990. Human-robot interaction via the transfer of power and information signals. IEEE Trans Syst Man Cybernet. 20(2):450-463.

Kazerooni H, Steger R, Huang L. 2003. Hybrid control of the Berkeley lower extremity exoskeleton. Int J Robot Res. 25(56):561-573.

Kirtley C. 2004. Automated diagnosis of gait abnormalities. Gait And Clinical Movement Analysis Society (GCMAS). Proceedings of the Annual Meeting of the Gait and Clinical Movement Analysis Society. 
Krebs HI, Hogan N, Aisen ML, Volpe BT. 2002. Robot-aided neurorehabilitation. IEEE Trans Rehabil Eng. 6(1):75-87.

McMillan AG, Kendrick K, Michael JW, Aronson JA, Horton GW. 2004. Preliminary evidence for effectiveness of a stance control orthosis. J Prosthet Orthot. 6:6-13.

Moreno JC, Brunetti F, Pons JL, Baydal JM, Barbera R. 2005. Rationale for Multiple compensation of muscle weakness walking with a wearable robotic orthosis. Paper presented at: International Conference on Robotics and Automation, Barcelona, Spain.

Moreno JC, Brunetti FJ, Rocon E, Pons J.L. 2008. Immediate effects of a controllable knee ankle foot orthosis for functional compensation of gait in patients with proximal leg weakness. Med Biol Eng Comput. 46(1):43-53.

Moreno JC, Rocon E, Ruiz AF, Brunetti F, Pons JL. 2006. Design and implementation of an inertial measurement unit for control of artificial limbs: application on leg orthoses. Sens Actuat B 118:333-337.
Perry J. Gait analysis: normal and pathological function. Thorofare, NJ: McGraw-Hill.

Pons JL. 2008. Wearable robots: biomechatronic exoskeletons, Chichester, UK: Wiley.

Pons JL, Forner-Cordero A, Rocon E, Moreno JC. 2008. Mechatronics and bioinspiration in actuator design and control, Appl Bion Biomech. 5(3):127-133.

Reinkensmeyer DJ, Emken JL, Cramer SC. 2004. Robotics, motor learning, and neurologic recovery. Annu Rev Biomed Eng. $6: 497-525$

Rocon E, Ruiz AF, Pons JL. 2008. Biomechanical modelling of the upper limb for robotics-based orthotic tremor suppression. Appl Bionics and Biomechanics 2(2):81-85.

Scott SH. 1999. Apparatus for measuring and perturbing shoulder and elbow joint positions and torques during reaching. $\mathbf{J}$ Neurosci Meth. 89(2):119-127.

Winter D. 1991. The biomechanics and motor control of human movement. Ontario, Canada: University of Waterloo. 

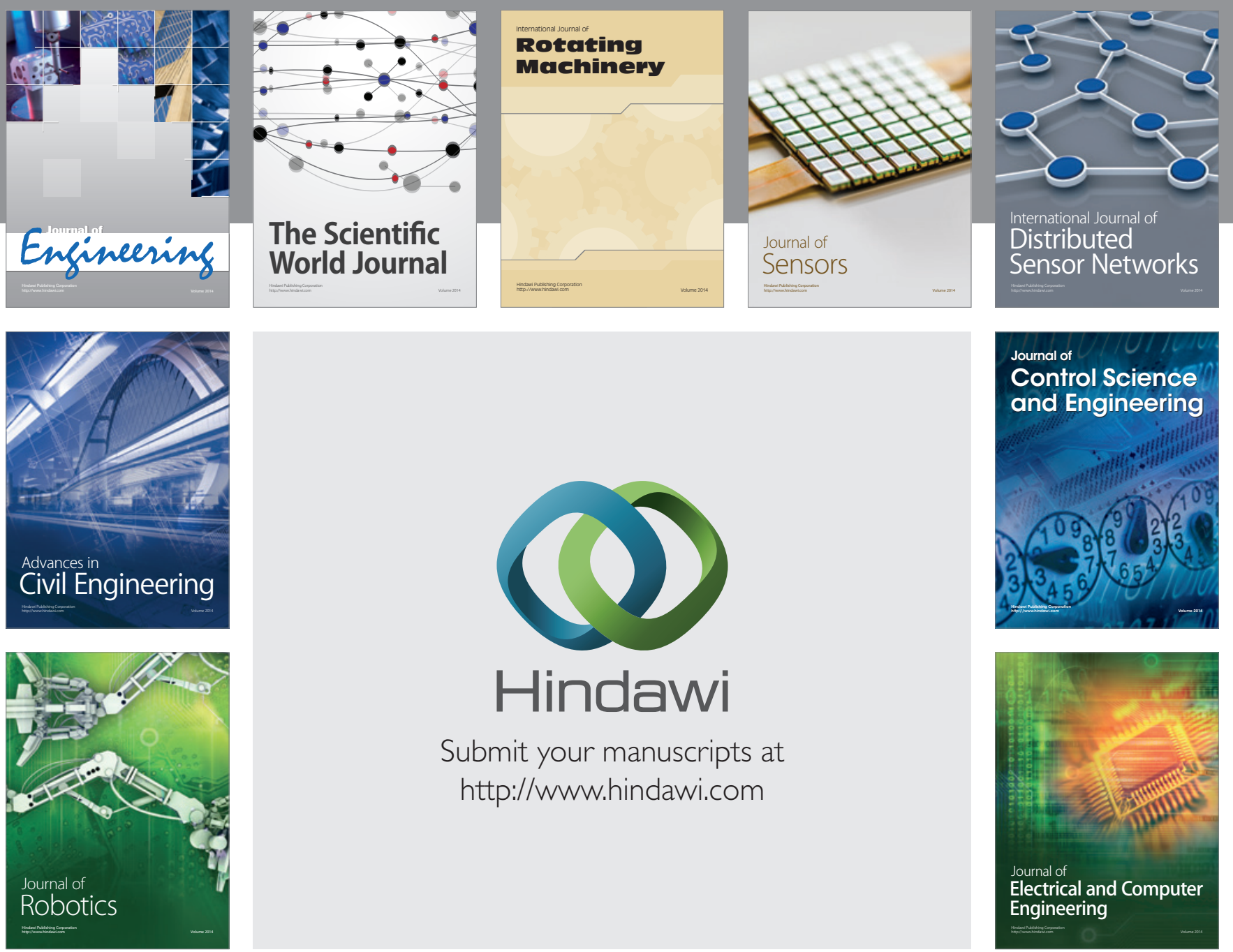

Submit your manuscripts at

http://www.hindawi.com
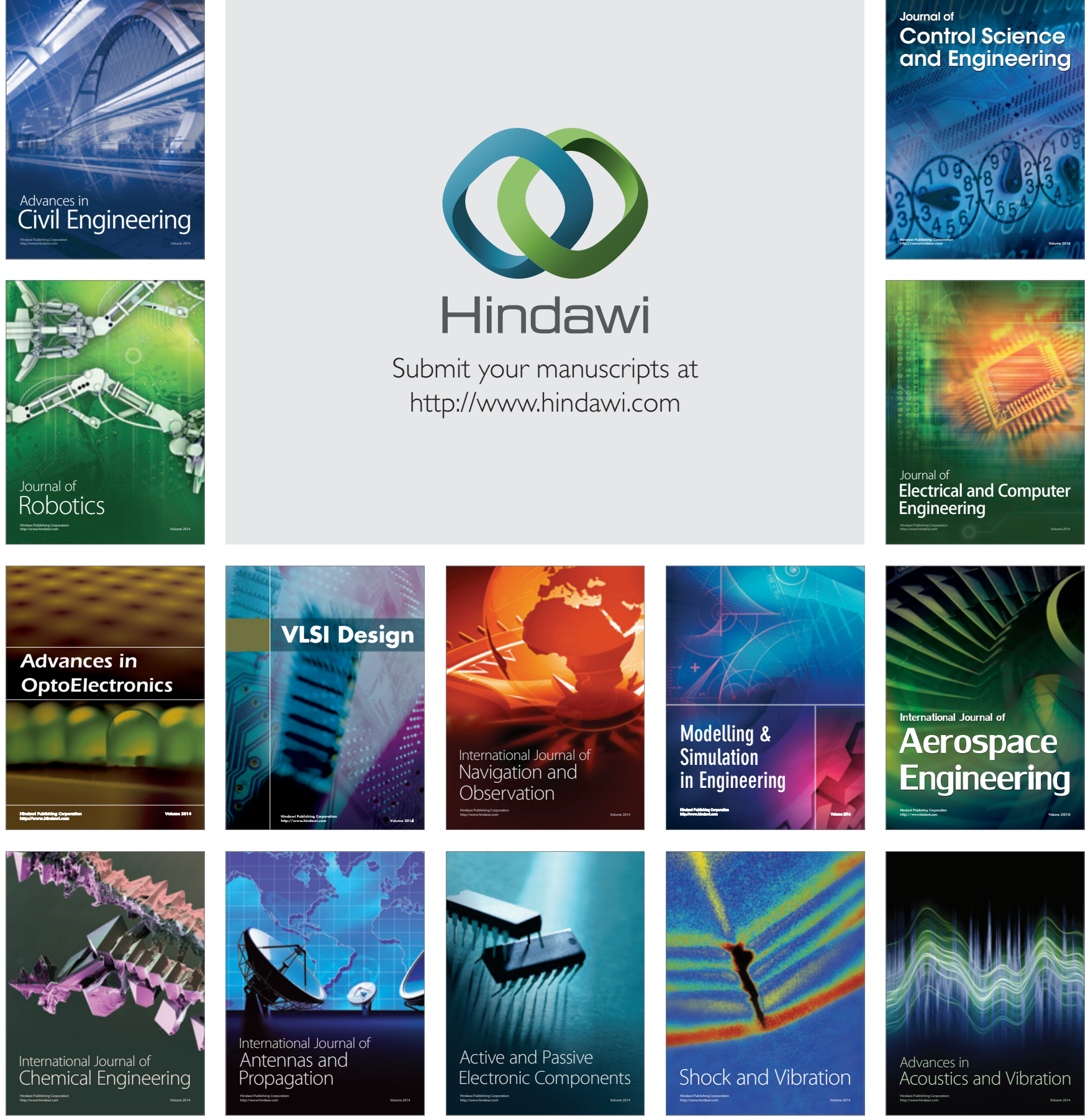